\title{
Bent crystals as a tool for manipulation of ultra-relativistic electron beams
}

\section{Bandiera ${ }^{1}$}

INFN Sezione di Ferrara

via Saragat 144122 Ferrara, Italy

E-mail: laura.bandieralcern.ch

E. Bagli, G. Germogli, V. Guidi, A . Mazzolari

INFN Sezione di Ferrara \& Università degli Studi di Ferrara, Italy

H. Backe, W. Lauth

Institut fur Kernphysik, Johannes Gutenberg Universitat Mainz, Germany

\section{A. Berra, D. Lietti, M. Prest}

INFN Sezione di Milano Bicocca \& Università degli Studi dell'Insubria, Italy

\section{De Salvador}

INFN Sezione di Legnaro \& Università degli Studi di Padova, Italy

\section{E. Vallazza}

INFN Sezione di Trieste, Italy

\section{Tikhomirov}

Institute for Nuclear Problem of Belarusian State University, Minsk, Belarus

Channeling and coherent interactions of charged particles in crystals are known since the 60s and used as a tool for material analysis by low-energy ion channeling and for the generation of linearly polarized $\gamma$-beams through coherent bremsstrahlung at electron accelerators. We report on an experiment carried out at the MAinzer MIkrotron with the aim of investigating the electromagnetic radiation generated by $855 \mathrm{MeV}$ electrons in a bent crystal. Such study allows one to investigate the influence of the crystalline curvature on the radiation emitted by sub-GeV electrons in a bent crystal and the possible application of this kind of radiation.

\section{'speaker}




\section{Introduction}

Channeling and coherent interaction of charged particles in crystals are known since the $60 \mathrm{~s}$ and used as a tool for material analysis by low-energy ion channeling and for the generation of linearly polarized $\gamma$-beams through coherent bremsstrahlung at electron accelerators $[1,2]$, such as the MAinzer MIcrotron (MAMI) in Germany and the Jefferson Lab in USA. Channeling consists in the trapping of charged particles with energy $E$ and with an angle of incidence with the crystal planes (axes) smaller than $\vartheta_{\text {critical }}=\sqrt{2 U_{0} / E}$ inside the planar (axial) potential well depth $\mathrm{U}_{0}$ [3].

If the crystal is bent, channeled particles follow the crystal bending and are steered from their initial direction. The usage of bent crystals has been deeply investigated for beam manipulation in hadron-accelerator, e.g., CERN SPS and LHC, where bent crystals can be exploited for beam steering in crystal-assisted collimation or extraction [4-7].

Bent crystals may found relevant application also for the manipulation of high-energy e \pm beams. In this case, beam deflection is accompanied by the generation of electromagnetic radiation.

Here, we present results on beam steering and, in particular, on intense e.m. radiation generation through interaction of high-energy electrons with bent crystals. In case of bent crystals, also un-channeled particles can be deflected through the mechanism of Volume Reflection (VR), which consists in the steering of over-barrier particle trajectories and occurs in a wide angular acceptance, which is equal to the bending angle of the crystal [8]. VR consists in the reversal of transverse momentum of over-barrier particles by the interaction with the planar potential barrier at the tangency point of the trajectory with curved crystalline planes. The experiment was carried out at MAMI with a sub-GeV electron beam. A particular attention is given to the possible applications, such as an intense crystal-based $\mathrm{x}$ - or $\gamma$-ray source.

\section{Experiment at MAMI}

A $855 \mathrm{MeV}$ electron beam, available at the MAMI B facility, was set at a beam size of $200 \times 70 \mu \mathrm{m}^{2}$ and a divergence of $70 \times 30 \mu \mathrm{rad}^{2}$ along the horizontal and vertical directions, respectively. In this way, the beam divergence resulted to be much less than the planar critical angle, being about $220 \mu \mathrm{rad}$ at this energy for the Si (111) planes.

The setup was based on the one of Ref. [9] with the adding of a single-sided microstrip Si detector developed at the INSULAB of the University of Insubria of Como and the INFN of Trieste (Italy). A bending magnet separates the photons emitted by the $855 \mathrm{MeV}$ electrons inside the crystal from the charged beam. The Si detector was placed $5.973 \mathrm{~m}$ downstream of the crystal along the electron beamline, with an angular resolution better than $10 \mu \mathrm{rad}$. At a distance of $8.627 \mathrm{~m}$ from the crystal along the photon beamline a 10 " x 10 " NaI scintillator detector. An aperture of $4 \mathrm{~mm}$ diameter in the lead shield surrounding the detector allows the collection of the forward portion of the emitted photons, resulting in a collimator aperture of $0.46 \mathrm{mrad}$, i.e., equal to $\sim 0.8$ times the typical radiation angle, $1 / \gamma \approx 600 \mu \mathrm{rad}$. More details on the experimental setup can be found in Ref. [10]. Finally, a (30.5 \pm 0.5$) \mu \mathrm{m}$ bent Si crystal fabricated at the Sensors and Semiconductor Laboratatory of Ferrara University, was used to steer the 855 electron beam. With the usage of a mechanical holder, a secondary bending radius of $33.5 \mathrm{~mm}$ 
of the (111) planes was obtained due to the quasi-mosaic effect [11]. The crystal holder was mounted on a high-precision goniometric system equipped with 5 degrees of freedom: two translational movements allow aligning the crystal on the horizontal and vertical directions with an accuracy of $1 \mu \mathrm{m}$; three rotational stages were used to rotate the sample around the $\mathrm{x}, \mathrm{y}$ and $\mathrm{z}$ axes with an accuracy of 50, 9 and $175 \mu \mathrm{rad}$, respectively. The crystal was positioned to deflect particles in vertical direction, i.e., normal to that of the bending magnet.

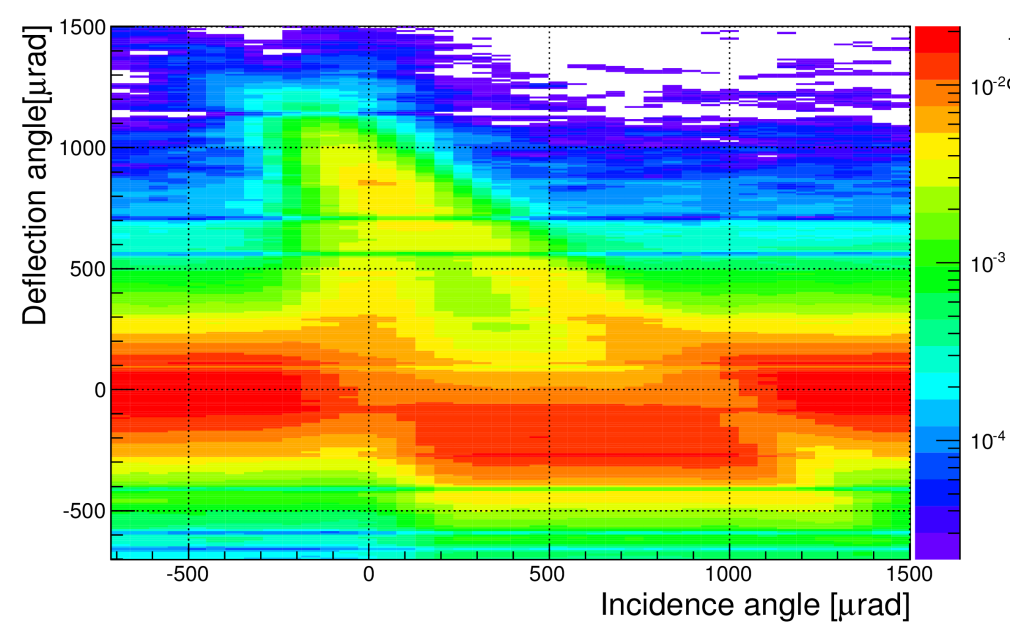

Figure 1. Experimental deflected beam distribution vs. the incoming angle with respect to the bent (111) planes.

We report here a brief summary of the deflecting proprieties of the crystal presented in Ref. [12]. The deflection angle and efficiency of the electrons vs. the angle between crystal planes with the beam were recorded. Fig. 1 represents the distribution of the beam particles after interaction with the crystal vs. the crystal-to-beam orientation. For incidence angle $\theta_{\text {in }}<-\theta_{\text {critical }}=$ $-220 \mu \mathrm{rad}$, the crystal planes are misaligned with respect to the beam direction and the crystals seems to scatter particles as an amorphous medium. For angle within two times the critical angle, particles are channeled and deflected at the crystal bending angle of about $910 \mu \mathrm{rad}$. For incidence angle larger than the critical one but smaller than the bending angle, $220 \mu \mathrm{rad}<\theta_{\text {in }}<$ $910 \mu \mathrm{rad}$, particles are volume-reflected by an angle of about $-190 \mu \mathrm{rad}$ in a direction opposite to that of crystal channeling. One should notice that at zero position not all the particles are deflected at the full bending angle, but there is a strong contribution of nearly undeflected particles or deflected at a smaller bending angle. Indeed, thus particles were dechanneled. Dechanneling is the kick out of particles from a channel as the transverse energy of channeled electrons becomes higher than the planar potential barrier due to the incoherent scattering of the particles by crystal electrons and nuclei. On the other hand, for the same reason, overbarrier particles may be captured by crystal planes; that occurs in the VR region, where some particles may be volume-captured in the planar potential well due to the contribution of incoherent scattering. Volume Capture (VC) consists in the entry of above-barrier particles into the channeling regime when particle trajectories are at the tangency point with the bent planes. 
Summarizing, it is evident from Fig. 1 that, even channeling of particle may give rise to a larger deflection angle, VR is much more efficient to steer a sub-GeV electron beam, since occur for overbarrier particles thus avoiding the spoiling effect of dechanneling.

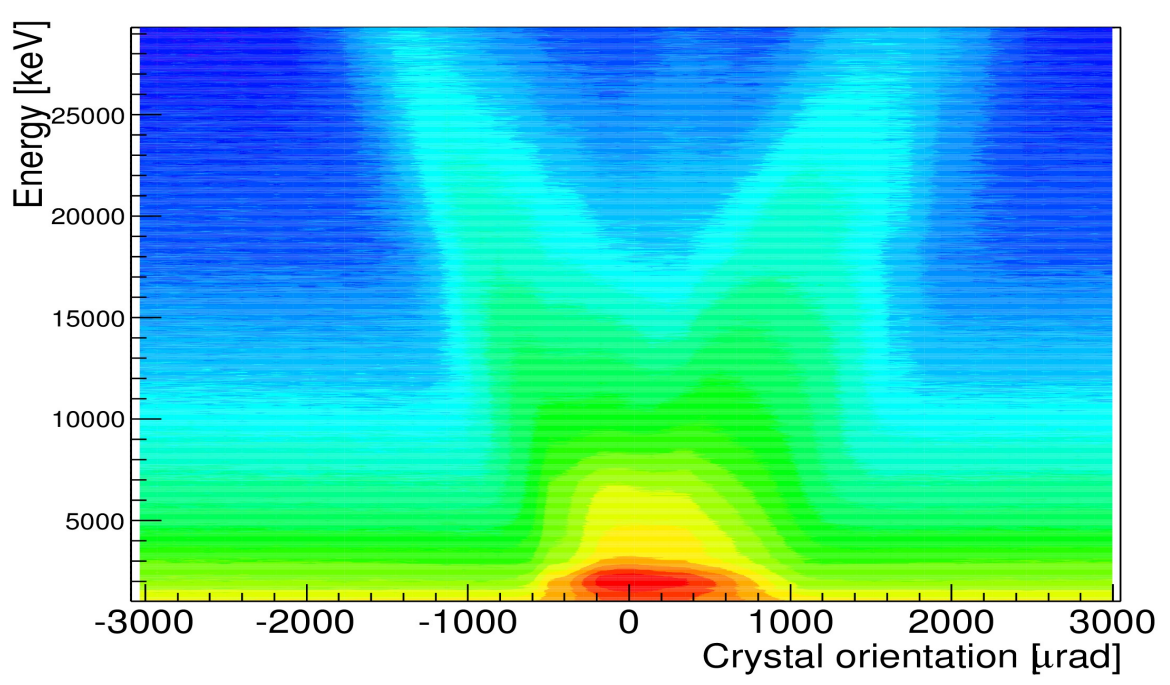

Figure 2. Experimental radiation spectrum vs. the incoming angle with respect to the bent (111) planes. The collimator aperture was $4 \mathrm{~mm}$, differently from Ref. [13] in which a larger aperture of $40 \mathrm{~mm}$ did not permitted to highlight the $\mathrm{CB} V$-tail structure caused by the forward photons.

Here, we report an investigation on the radiation emitted by $855 \mathrm{MeV}$ electrons in the bent crystal in case of alignment and misalignment between the beam direction and the bent (111) Si planes[13]. Fig. 2 displays the recorded experimental radiation spectrum after interaction with the crystal vs. the crystal-to-beam orientation: the angular range selected lies within -3 and 3 mrad. One may notice the low energy peak, which are associated with planar channeling at zero incidence, splits at increasing photon energy into two well distinguishable branches with enhanced photon intensity (see the $V$ pattern in the picture). This phenomenon is observed just in the transition region between channeling radiation (CR) and coherent bremsstrahlung (CB) emission. We remind here that $\mathrm{CR}$ is generated by the nearly oscillatory motion of electrons in the planar channels, while CB peaks are generated by overbarrier electrons/positrons crossing the periodical structure of a crystal lattice, i.e., crossing crystal planes with a small incidence angle but still larger than $\theta_{\text {critical }}$. CB radiation is expected to be harder due to the smaller oscillatory period, which increases with $\theta_{\text {in }}$, but less intense tha CR. The $V$-shape feature has already been reported in the literature for straight crystals (see for instance Fig. 12 in [9]), while is a novelty for the case of bent crystals, since the collimator apeture used for the goniometer scan presented in Ref. [13] was too large, $40 \mathrm{~mm}$, to highlight the $\mathrm{CB} V$-tail structure, caused by the forward photons. The usage of a smaller aperture permitted also to visualize better the loss of intensity for hard photon emission under CB.

In Fig. 2, one may also notice the extension of the intense and soft CR zone to the VR region on the right side of zero incidence angle. Indeed, for straight crystals, the radiation 
intensity falls off very rapidly out of the channeling region, which is as large as $2 \theta_{\text {critical }}=440$ $\mu \mathrm{rad}$. The strongest intensity for VR as compared to $\mathrm{CB}$, that makes this kind of radiation more similar to CR, has been explained in Ref. [13] by the contribution of volume-captured particles, which have a similar dynamics to channeled ones.

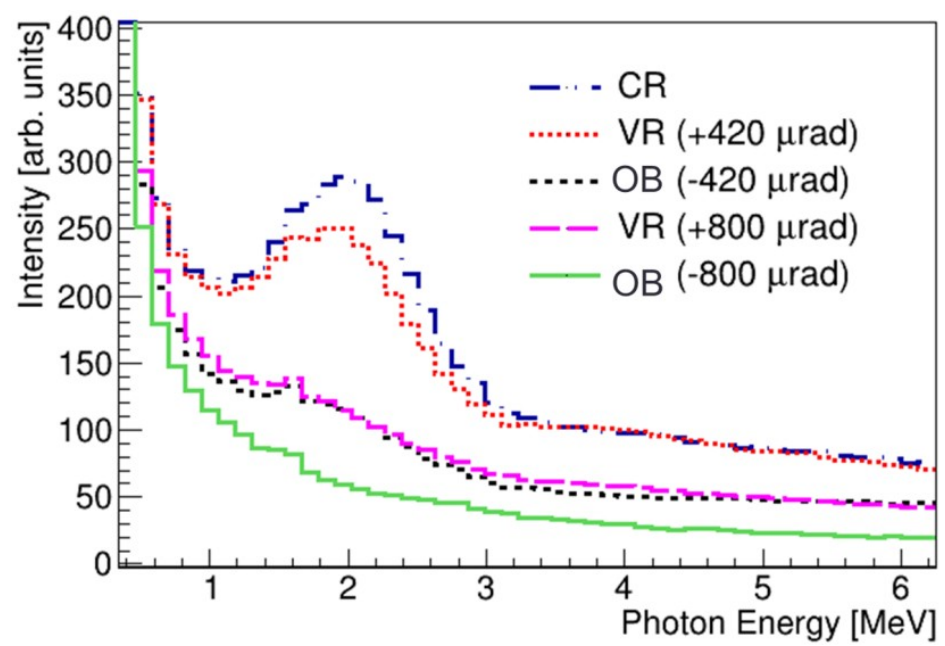

Figure 3. Experimental spectra for channeling (blue), VR at $\theta_{\text {in }}=420 \mu \mathrm{rad}$ (red) and at $\theta_{\text {in }}=800$ $\mu \mathrm{rad}$ (pink) from perfect alignment with the bent (111) Si planes. For comparison also the over-barrier (OB) spectra at at $\theta_{\text {in }}=-420 \mu \mathrm{rad}$ (black) and at $\theta_{\text {in }}=-800 \mu \mathrm{rad}$ (green) are shown.

For a more detailed investigation of the features of the radiation accompanying VR, Fig. 3 shows the single spectra collected at different crystal-to-beam orientations: blue line for zero incidence angle (CR), at $\theta_{\text {in }}=+420 \mu \mathrm{rad}$ (red line) in the middle of VR region and at $\theta_{\text {in }}=800$ $\mu \mathrm{rad}$ (pink line) at the end of $\mathrm{VR}$ region. In the latter case the contribution of $\mathrm{VC}$ is weaker, because electrons can be captured only at the crystal end, and thereby the radiation peak is less pronounced. In all these three cases, the radiation is peaked around $1.8 \mathrm{MeV}$, being the peak more pronunced for $\mathrm{CR}$ and for VR at $\theta_{\text {in }}=+420 \mu \mathrm{rad}$. In order to compare VR with the misalignment case, Fig. 3 also shows the spectra at $\theta_{\text {in }}=-420 \mu$ rad (black line) on the opposite side to VR region and at $\theta_{\text {in }}=-800 \mu \mathrm{rad}$ (green line). One may notice that the radiation accompanying VR remains more intense than for the over-barrier case with opposite incidence angle to crystal planes.

\section{Conclusions}

The radiation emitted by $855 \mathrm{MeV}$ electrons via planar channeling and volume reflection in a tens-micrometer long bent $\mathrm{Si}$ crystal was recorded. In both cases, the radiation spectra were much more intense than for the case of misalignment between crystal planes and beam direction, i.e. than for an equivalent amorphous material. Furthermore, the radiation spectra were peaked in the $\mathrm{MeV}$ energy range.

As already seen in the $100 \mathrm{GeV}$ energy range [14,15], the deflection efficiency is larger for VR than for channeling and the angular acceptance for both radiation and beam deflection is wider. Indeed, here we showed that the radiation accompanying VR is comparable in intensity to the radiation in straight crystals, i.e., it is a sort of intermediate case between CR and CB. Indeed, the intensity of radiation accompanying volume reflection is maintained high in the whole 
angular acceptance, thus making this effect a good candidate for the realization of a gamma-ray source with poor-emittance electron beams in the Sub-GeV/GeV energy range accessible by many electron accelerators worldwide, e.g. SLAC, MAMI and JLab.

\section{References}

[1] M. L. Ter-Mikaelian, High-Energy Electromagnetic Processes in Condensed Media (Wiley, New York, 1972).

[2] V. Baier, V. Katkov, and V. Strakhovenko, Electromagnetic Processes at High Energies in Oriented Single Crystals (World Scientific, Singapore, 1998).

[3] J. Lindhard, Danske Vid. Selsk. Mat. Fys. Medd. 34, 14361 (1965).

[4] E. Tsyganov, Tech. Rep. (Fermilab, 1976) preprint TM-682.

[5] V. M. Biryukov et al., Nucl. Instrum. Methods Phys. Res. Sect. B 234, 23 (2005).

[6] E. Uggerhoj and U. Uggerhoj, Nucl. Instrum. Methods Phys. Res. Sect. B 234, 31 (2005).

[7] W. Scandale et al., Phys. Lett. B 692, 78 (2010).

[8] A. Taratin and S. Vorobiev, Phys. Lett. A 119, 425 (1987).

[9] H. Backe, P. Kunz, W. Lauth, and A. Rueda, Nucl. Instrum. Methods Phys. Res., Sect. B 266, 3835 (2008).

[10] D. Lietti et al., Rev. Sci. Instrum. 86, 045102 (2015).

[11] G. Germogli, A. Mazzolari, L. Bandiera, E. Bagli, and V. Guidi, Nucl. Instrum. Methods Phys. Res., Sect. B 355, 81 (2015).

[12] A. Mazzolari et al., Phys. Rev. Lett. 112, 135503 (2014).

[13] L. Bandiera et al., Phys. Rev. Lett.115, 025504 (2015).

[14] W. Scandale et al., Phys. Rev. A 79, 012903 (2009).

[15] L. Bandiera et al., Nucl. Instrum. Methods Phys. Res., Sect. B 309, 135 (2013). 